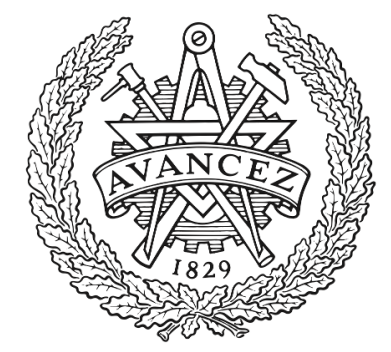

CHALMERS

UNIVERSITY OF TECHNOLOGY

\title{
The Far-Field Equatorial Array for Binaural Rendering
}

Downloaded from: https://research.chalmers.se, 2023-04-26 13:21 UTC

Citation for the original published paper (version of record):

Ahrens, J., Helmholz, H., Alon, D. et al (2021). The Far-Field Equatorial Array for Binaural

Rendering. ICASSP, IEEE International Conference on Acoustics, Speech and Signal Processing -

Proceedings, 2021-June: 421-425. http://dx.doi.org/10.1109/ICASSP39728.2021.9414368

N.B. When citing this work, cite the original published paper. 


\section{THE FAR-FIELD EQUATORIAL ARRAY FOR BINAURAL RENDERING}

\author{
Jens Ahrens, Hannes Helmholz* \\ Chalmers University of Technology \\ 41296 Gothenburg, Sweden \\ first.lastechalmers.se
}

\author{
David Lou Alon, Sebastià V. Amengual Garí \\ Facebook Reality Labs Research \\ Facebook, 1 Hacker Way, Menlo Park, CA 94025 \\ \{davidalon, samengual\} @fb.com
}

\begin{abstract}
We present a method for obtaining a spherical harmonic representation of a sound field based on a microphone array along the equator of a rigid spherical scatterer. The two-dimensional plane wave decomposition of the incoming sound field is computed from the microphone signals. The influence of the scatterer is removed under the assumption of distant sound sources, and the result is converted to a spherical harmonic (SH) representation, which in turn can be rendered binaurally. The approach requires an order of magnitude fewer microphones compared to conventional spherical arrays that operate at the same $\mathrm{SH}$ order at the expense of not being able to accurately represent non-horizontally-propagating sound fields. Although the scattering removal is not perfect at high frequencies at low harmonic orders, numerical evaluation demonstrates the effectiveness of the approach.
\end{abstract}

Index Terms - Microphone array, binaural rendering, HRTFs, spherical harmonics, plane wave decomposition

\section{INTRODUCTION}

Spherical microphone arrays have been shown to be a convenient solution for capturing spatial sound fields when equal properties of the method are desired for all angles of sound incidence [1, 2, 3, 4]. Arrays that comprise a rigid spherical scatterer on which the microphones are placed are most convenient. Representing the captured sound field in terms of spherical harmonics $(\mathrm{SH})$ has shown to be a flexible representation that enables applications like beamforming [4] and binaural rendering [5]. Binaural rendering computes the signals that would arise at the ears of a person when their head is exposed to the sound field captured by the array. This will be the targeted application in our work. It requires the user's head-related transfer functions (HRTFs) and can be performed either directly in the SH domain [5] or by sampling of the plane wave decomposition (PWD) of the sound field [6] by means of beamforming.

When it comes to capturing or reproducing sound scenes for virtual reality, there may be scenarios in which it may be sufficient to restrict the considerations to the horizontal plane $[7,8]$ because this is the most common real-world scenario, and the human auditory system is optimized for it [9]. Circular microphone arrays with appropriate scatterers therefore exhibit considerable potential in this context as they may achieve the same spatial resolution with an order of magnitude fewer microphones.

Cylindrical scatterers with a circular microphone array along the circumference and partly also with additional microphones were investigated in $[4,7,10,11,12,13,14]$. The evaluation was performed mostly with respect to beamforming performance and sound source

\footnotetext{
${ }^{*}$ We thank Facebook Reality Labs for funding the presented work.
}

localization capabilities. The evaluation in [14] focused on the accuracy of the extracted sound field representation. [12, 15, 16] investigate beamforming based on a circular array along the equator of a spherical scatterer. We will assume such a setup in the remainder and refer to it as equatorial array.

$\mathrm{SH}$ decomposition based on a circular array on a planar scatterer was presented in [17] and based on concentric circular arrays without a scatterer in [18]. [19] used an equatorial microphone array for resynthesis of the sound field using a circular loudspeaker array using a formulation that was partially in $\mathrm{SH}$.

In [20], a method for binaural rendering of equatorial array signals without sound field decomposition is presented. Head tracking is possible only along the azimuth, and the array radius has to match the listener's head radius. We aim at an SH representation of the captured sound field for being able to apply tracking of the user's head orientation with respect to arbitrary rotations.

\section{METHOD}

\subsection{A Single Plane Wave}

Assume that a plane wave with a propagation direction $(\phi, \theta)=$ $(\pi / 2, \theta)$ that is parallel to the horizontal plane impinges on a rigid spherical scattering body in a free-field. $\phi$ denotes the colatitude and $\theta$ the azimuth in a spherical coordinate system. The scattering body is centered at the coordinate origin. The sound pressure field $S_{\mathrm{pw}}^{\text {surf }}(\vec{x}, \omega)$ of radian frequency $\omega$ at location $\vec{x}$ on the surface of the scatterer is symmetrical w.r.t. rotation about the propagation vector through the coordinate origin. Cf. Fig. 1 (left). Therefore, if the sound pressure due to the plane wave is known on the equator, we can compute the sound pressure everywhere on the sphere.

A plane wave that propagates into direction $\theta$ "hits" the scattering object first at position $(\pi / 2, \theta+\pi)$ on the equator. The central angle $\Delta \sigma(\beta, \alpha, \theta)$ between the first intersection point and an arbitrary position $(\beta, \alpha)$ on the sphere can be determined via the spherical law of cosines to be

$$
\Delta \sigma(\beta, \alpha, \theta)=\pi-\arccos (\sin \beta \cos (\theta-\alpha)) .
$$

Cf. Fig. 1 (right). We can therefore conclude that the sound pressure $S_{\mathrm{pw}}^{\text {surf }}(\vec{x}, \omega)$ that the considered plane wave produces at location $(\beta, \alpha)$ is identical to the sound pressure at the point $(\pi / 2, \theta+\pi \pm \Delta \sigma(\beta, \alpha, \theta))$. We choose $-\Delta \sigma$ and omit the dependency on the three angles in the notation for convenience.

\subsection{Computation of the Sound Pressure Distribution}

We need to apply the following 3 steps in order for being able to exploit the symmetries that are illustrated in Fig. 1 with arbitrary horizontally propagating sound fields $S(\vec{x}, \omega)$ : 

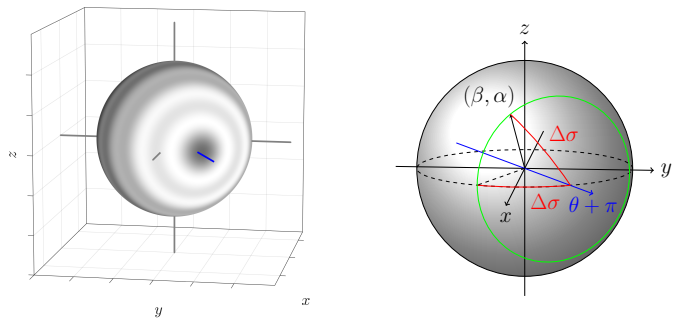

Fig. 1: Symmetry of the sound pressure on the sphere: The plane wave propagates horizontally into direction $\theta$, which is parallel to the blue line. The sound pressure is constant everywhere along the green circle (right plot).

1) Use the microphones along the equator to decompose the impinging sound field into a continuum of horizontally propagating plane waves and undo the effect of the scatterer. 2) Exploit the rotational symmetry of the sound field of plane waves on the scattering object to determine the sound pressure at the location of interest for the considered plane wave component. 3) Integrate over the continuum of plane waves for the location of interest to obtain the total sound pressure.

We employ a two-dimensional (2D) PWD defined as [4]

$$
S(\vec{x}, \omega)=\frac{1}{2 \pi} \int_{0}^{2 \pi} \bar{S}(\theta, \omega) \mathrm{e}^{-\mathrm{i} \frac{\omega}{c} r \cos (\theta-\alpha)} \mathrm{d} \theta,
$$

whereby $r$ is the radius, $c$ is the speed of sound, and $\bar{S}(\theta, \omega)$ are the coefficients of the PWD given by

$$
\bar{S}(\theta, \omega)=\sum_{m=-\infty}^{\infty} \mathrm{i}^{m} \breve{S}_{m}(\omega) \mathrm{e}^{\mathrm{i} m \theta}
$$

The circular harmonic $(\mathrm{CH})$ coefficients $\breve{S}_{m}(\omega)$ are computed from integrating $S(\vec{x} \mid, \omega)$ along a circular contour under free-field conditions using $[4,21]$

$$
\breve{S}_{m}(\omega) J_{m}\left(\omega \frac{r}{c}\right)=\stackrel{\circ}{S}_{m}(r, \omega)=\frac{1}{2 \pi} \int_{0}^{2 \pi} S\left(\left.\vec{x}\right|_{r}, \omega\right) \mathrm{e}^{-\mathrm{i} m \alpha} \mathrm{d} \alpha
$$

and can represent the sound pressure field $S(\vec{x}, \omega)$ via

$$
S(\vec{x}, \omega)=\sum_{m=-\infty}^{\infty} \breve{S}_{m}(\omega) J_{m}\left(\omega \frac{r}{c}\right) \mathrm{e}^{\mathrm{i} m \alpha} .
$$

$\alpha$ the azimuth in the polar coordinate system, and $J_{m}(\cdot)$ denotes the Bessel function of order $m$. In the presence of an acoustically rigid cylinder of radius $R$ that is centered at the $z$ axis, $J_{m}(\cdot)$ in (5) has to be replaced with $D_{m}(\cdot)$ given by [10]

$$
D_{m}(r, \omega)=J_{m}\left(\omega \frac{r}{c}\right)-\frac{J_{m}^{\prime}\left(\omega \frac{R}{c}\right)}{H_{m}^{\prime(2)}\left(\omega \frac{R}{c}\right)} H_{m}^{(2)}\left(\omega \frac{r}{c}\right),
$$

to account for the scattering. $H_{m}^{(2)}(\cdot)$ are the Hankel functions and the prime ' denotes differentiation with respect to the argument.

The placement of the microphones allows for approximating the integral in (4) (with (6)) along $r=R$, which yields the $\mathrm{CH}$ coefficients of the sum of incident and scattered sound field on the surface of the scatterer. We term the coefficients $S_{m}^{\text {surf }}(R, \omega)$.

$2 \mathrm{D}$ representations like (4) and (5) are very useful in the present context. However, they cannot describe 3D scattering scenarios but
2D ones such as the scattering off an infinitely long acoustically rigid cylinder. The scattering that we observe on the surface of our spherical scattering object will be different to the scattering due to a cylinder. Fortunately, there is a close relation between spherical and cylindrical scattering. Conversions between 2D and 3D representations have been presented in [22, 23, 24] but these cannot be exploited in the present context. We demonstrate in the Appendix that the modal correction term $X_{m}(R, \omega)$ given by (15) allows for representing the spherical scattering due to plane waves in a $\mathrm{CH}$, i.e. a 2D, representation so that the free-field PWD coefficients $\bar{S}(\theta, \omega)$ of the incoming sound field can be computed from (3) and (17). Note that this solution is only applicable for far-field sources.

We use (1) and (11) to obtain the sound pressure $S_{\mathrm{pw}}^{\mathrm{surf}}(\beta, \alpha, R, \omega)$ due to a single horizontally propagating unit-amplitude plane wave at an arbitrary location - given by the colatitude $\beta$ and azimuth $\alpha-$ on the surface of the spherical scatterer as

$$
\begin{gathered}
S_{\mathrm{pw}}^{\text {surf }}(\beta, \alpha, R, \omega)=S_{\mathrm{pw}}^{\text {surf }}(\pi / 2, \theta+\pi-\Delta \sigma, R, \omega) \\
=\sum_{\substack{n=|m| \\
m=-\infty}}^{\infty} 4 \pi \mathrm{i}^{-n}\left[Y_{n}^{m}(\pi / 2,0)\right]^{2} d_{n}(R, \omega) \mathrm{e}^{-\mathrm{i} m \theta} \mathrm{e}^{\mathrm{i} m(\theta+\pi-\Delta \sigma)} \\
=\sum_{m=-\infty}^{\infty} \mathrm{i}^{m} Z_{m}(R, \omega) \mathrm{e}^{-\mathrm{i} \Delta \sigma}
\end{gathered}
$$

whereby $Y_{n}^{m}(\cdot), d_{n}(R, \omega)$, and $Z_{m}(R, \omega)$ are defined in the Appendix by (12), (13) and (15), respectively.

To obtain the total sound pressure $S^{\text {surf }}(\beta, \alpha, R, \omega)$ at the location of interest on the surface of the spherical scatterer, we apply (2), whereby we weight the free-field PWD coefficients $\bar{S}(\theta, \omega)$ with the pressure $S_{\mathrm{pw}}^{\text {surf }}(\cdot)$ that each plane wave evokes on the scatterer as

$$
\begin{aligned}
& S^{\text {surf }}(\beta, \alpha, R, \omega)=\frac{1}{2 \pi} \int_{0}^{2 \pi} \bar{S}(\theta, \omega) S_{\mathrm{pw}}^{\text {surf }}(\beta, \alpha, R, \omega) \mathrm{d} \theta \\
= & \sum_{m^{\prime}=-\infty}^{\infty} \mathrm{i}^{m^{\prime}} \breve{S}_{m^{\prime}}(\omega) \sum_{m=-\infty}^{\infty} \mathrm{i}^{m} Z_{m}(R, \omega) \frac{1}{2 \pi} \int_{0}^{2 \pi} \mathrm{e}^{\mathrm{i} m^{\prime} \theta} \mathrm{e}^{-\mathrm{i} m \Delta \sigma} \mathrm{d} \theta .
\end{aligned}
$$

Recall that $\breve{S}_{m^{\prime}}(\omega)$ can be computed from the microphone signals using (17). The integral in (8) has to be evaluated numerically, which is straightforward as it is dependent neither on frequency nor on the array geometry.

\subsection{Computation of the Spherical Harmonic Coefficients}

We compute the free-field SH coefficients $\breve{S}_{n}^{m^{\prime \prime}}(\omega)$ of the captured sound field $S(\beta, \alpha, R, \omega)$ from (8) using the standard transformation integral that integrates the sound pressure over the surface of the spherical scatterer as $[3,21]$

$$
\begin{aligned}
& \breve{S}_{n}^{m^{\prime \prime}}(\omega)=\frac{1}{d_{n}(R, \omega)} \oint_{O} S^{\text {surf }}(\beta, \alpha, R, \omega) Y_{n}^{m^{\prime \prime}}(\beta, \alpha)^{*} \mathrm{~d} \Omega \\
& =\frac{1}{d_{n}(R, \omega)} \sum_{m^{\prime}=-\infty}^{\infty} \mathrm{i}^{m^{\prime}} \breve{S}_{m^{\prime}}(\omega) \sum_{m=-\infty}^{\infty} \mathrm{i}^{m} Z_{m}(R, \omega) \\
& \quad \times(-1)^{m^{\prime \prime}} \sqrt{\frac{2 n+1}{4 \pi} \frac{\left(n-\left|m^{\prime \prime}\right|\right) !}{\left(n+\left|m^{\prime \prime}\right|\right) !}} \\
& \quad \times \frac{1}{2 \pi} \int_{0}^{2 \pi} \oint_{O} \mathrm{e}^{\mathrm{i} m^{\prime} \theta} \mathrm{e}^{-\mathrm{i} m \Delta \sigma} P_{n}^{\left|m^{\prime \prime}\right|}(\cos \beta) \mathrm{e}^{-\mathrm{i} m^{\prime \prime} \alpha} \mathrm{d} \Omega \mathrm{d} \theta .
\end{aligned}
$$


The spherical harmonics $Y_{n}^{m}(\cdot)$ are defined in (12). The asterisk * denotes the complex conjugate. $\mathrm{d} \Omega$ is an infinitesimal spherical surface element, $O$ denotes the unit sphere. $d_{n}(R, \omega)$ is defined by (13), and dividing through it in (9) removes the effect of the scatterer.

The integral in (9) may be interpreted as an integral over the volume of a sphere and can be evaluated using standard quadrature. It is independent of frequency and of the array geometry and can be pre-computed. The infinite summations in above equations, particularly (8), (9), and (15), need to be bounded in practice to a maximum order $N$ such that $n,|m| \leq N$.

\section{RESULTS AND DISCUSSION}

\subsection{Accuracy of the Sound Field Estimation}

Fig. 2 (top) depicts a cylindrical projection of the sound pressure on the surface of a rigid sphere of radius $R=0.1 \mathrm{~m}$ due to a monochromatic horizontally propagating plane wave of $f=1 \mathrm{kHz}$ and maximum order $N=8$. This is our reference sound field that we would like to compute solely from knowledge of the sound pressure along the equator. The same sound field estimated via (8) using a dense equatorial array is depicted in Fig. 2 (middle). The difference between the two is depicted in Fig. 2 (bottom).

Next, we compare the accuracy of the proposed equatorial array to a conventional spherical array of the same order $N=8$. Cf. Fig. (3) for an illustration of the geometry. Mind the different amounts of microphones (110 vs. 17). We let both arrays capture a plane wave and use the obtained 8th-order coefficients to estimate the sound pressure at a dense grid of locations on the spherical scattering object using (8). We evaluate the accuracy of the estimation using the average relative error $E(\omega)$ given by

$$
E(\omega)=\frac{1}{L} \sum_{l}\left|\left(\hat{S}\left(\vec{x}_{l}, \omega\right)-S\left(\vec{x}_{l}, \omega\right)\right) / S\left(\vec{x}_{l}, \omega\right)\right|,
$$

whereby $\hat{S}\left(\vec{x}_{l}, \omega\right)$ denotes the sound pressure at each of the $L$ evaluated locations $\vec{x}_{l}$ as estimated by one of the arrays, and $S\left(\vec{x}_{l}, \omega\right)$ denotes the true sound pressure. $E(\omega)$ is depicted in Fig. 4 for a plane propagating into different elevations. The left-most parts of each subplot represent a horizontally propagating plane wave, for which the equatorial array shows a slightly larger error compared to the conventional spherical array below $5 \mathrm{kHz}$ (see the inset). Neither array can capture the plane wave accurately above $5 \mathrm{kHz}$ because of order truncation and spatial aliasing.

The accuracy of the conventional array is independent of the elevation whereas the proposed array is not capable of reconstructing the sound pressure for propagation directions outside of the horizontal plane. This is expected as the proposed array assumes horizontally propagating sound fields.

\subsection{Binaural Rendering}

Our target application is binaural rendering the detailed evaluation of which is beyond the scope of this paper. We present a first analysis here to indicate the potential.

Fig. 5 depicts the difference between the binaural signals produced by the two arrays from Fig. 3 for a plane wave that impinges from different elevations. The rendering was performed directly in the SH-domain based on the coefficients computed from (9) and using the implementation from [25], which is available in [26]. It can be seen that systematic differences arise above a few hundred $\mathrm{Hz}$. The differences may be partly attributed to the circumstance that the equatorial array always produces a horizontally propagating sound

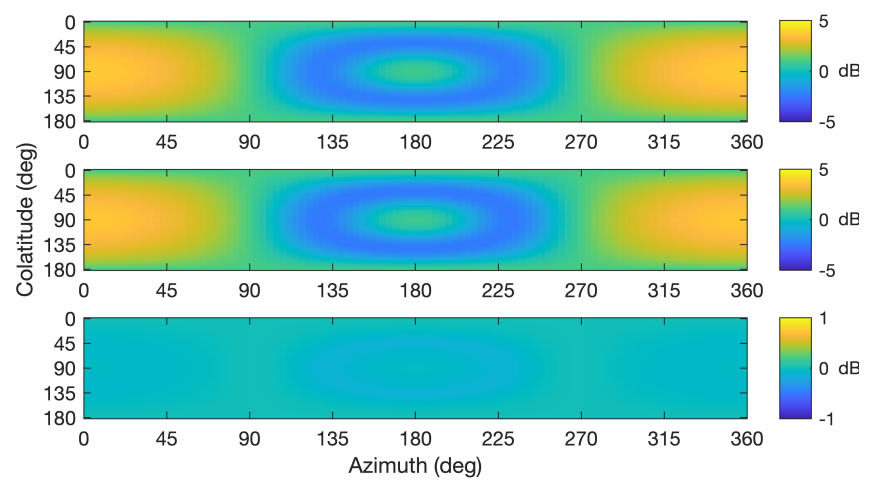

Fig. 2: Top: Reference sound pressure distribution on a logarithmic scale. Middle: sound pressure reconstructed from a dense equatorial array according to Eq. (8). Bottom: Difference between the two. Mind the different color scales.
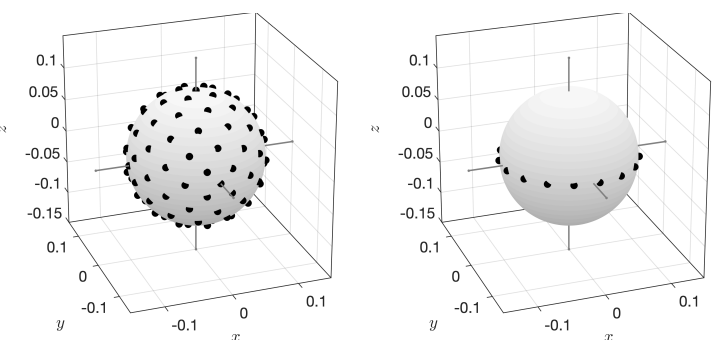

Fig. 3: Illustration of the microphone array geometries. Both support a maximum order of $N=8$. Left: 110-node Lebedev grid. Right: $2 N+1=17$-node equatorial grid. The black dots denote the locations of the microphones.
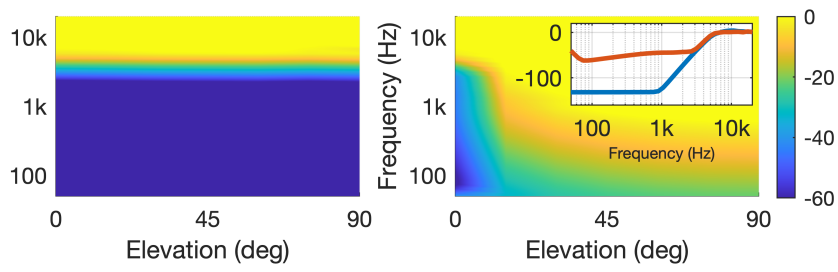

Fig. 4: $20 \log _{10}|E(\omega)|$ according to Eq. (10) for a plane wave that impinges from different elevations that is captured by the two microphone arrays from Fig. 3. Left: the conventional spherical array. Right: proposed equatorial array. The inset depicts $20 \log _{10}|E(\omega)|$ for the horizontally propagating plane wave for the spherical array (blue) and the equatorial array (red) in more detail.

field so that certain elevation dependent HRTF cues are not triggered. The binaural signals from the equatorial array for horizontally propagating plane waves show also higher magnitudes above approx. $4 \mathrm{kHz}$. This may be attributed to the imperfect scattering removal (cf. Sec. 3.3).

Note that the data depicted in Fig. 5 were computed from 3rdoctave smoothed signals. With unsmoothed data, the shift of the center frequency of a notch that may be apparent in the magnitude spectrum leads to large differences that make it difficult to deduce general trends and the perceptual relevance of which might be low.

We provide static (non-head-tracked) audio examples in [27] for a variety of different sound fields including elevated sound sources. Informal listening confirms that the rendering works well if the cap- 


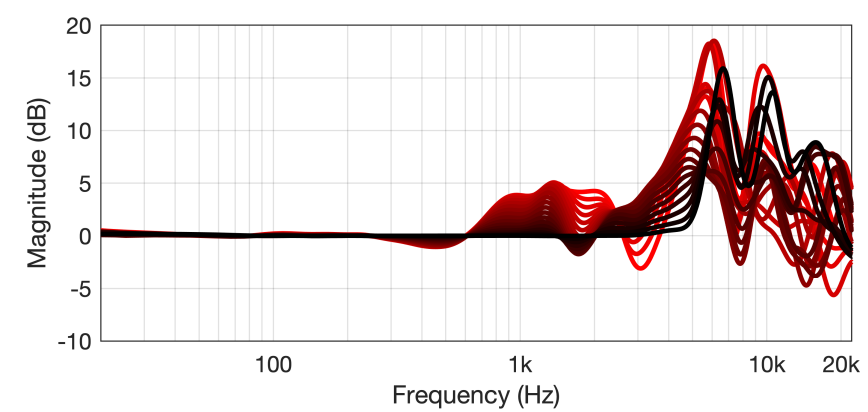

Fig. 5: Difference between the binaural signals produced by the two arrays from Fig. 3 for a plane wave impinging from different elevations ranging from $0^{\circ}$ (black) to $90^{\circ}$ (red) in steps of $5^{\circ}$.
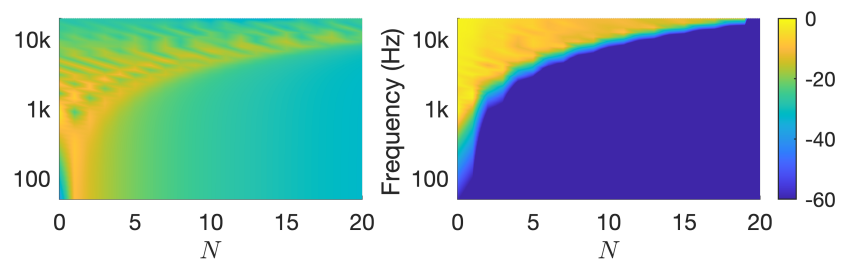

Fig. 6: $20 \log _{10}|E(\omega)|$ according to (10) between the sound field on a cylindrical scatterer according to (5) and (6) and on the equator of a spherical scatterer according to (11) for a horizontally propagating plane wave as a function of the maximum included harmonic or$\operatorname{der} N$. Left: no correction. Right: with correction according to (15).

tured sound field fulfills the assumptions. Violations of the underlying assumptions such as non-horizontal propagation do not render void the approach.

\subsection{Discussion}

Because of the mathematical formulation, the output of the array is necessarily a horizontally propagating sound field. Even if the captured sound field is propagating only horizontally, deviations occur as evident from Fig. 2, 4, and 6. The reason for this lies in the scattering removal represented by (17). The correction via $Z_{m}(\cdot)$ is exact at infinite orders. For finite orders, the scattering correction is very accurate at low frequencies as evident from Fig. 6 (right), which compares the left-hand side and the right-hand side of (14) for different maximum orders $(n,|m|) \leq N$. Note that the 2D expansion and the 3D expansion in (14) exhibit different radial dependency. We will investigate this aspect in future work.

Despite the limitations of the scattering removal, the available data suggest that the presented approach produces a lower error for horizontally propagating sound fields compared to the cylindrical array from [14], which was composed of multiple circular rings of microphones with total number of microphones that was equal to a conventional spherical array of the same SH order. The circumstance that typical arrays are generally not capable of capturing the sound field accurately at high frequencies has been shown to be tolerable particularly when binaural rendering is the targeted application [28].

\section{CONCLUSIONS}

We presented a method for spherical harmonic decomposition of a sound field composed of distant sound sources based on a circular microphone array along the equator of a spherical scatterer. A first evaluation suggests that the proposed solution can be useful for binaural rendering applications.

We will present a new solution to the problem that does not put any requirements on the source distance in [29].

\section{APPENDIX: EXPRESSING 3D SCATTERING USING A 2D REPRESENTATION}

We demonstrate how to undo the scattering due to the spherical object in the 2D representation (5) to obtain the free-field CH coefficients $\breve{S}_{m}(\omega)$ of the impinging sound field.

In general, the sound field $S_{\mathrm{pw}}^{\text {surf }}(\beta, \alpha, R, \omega)$ on the surface of a spherical scattering object with radius $R$ due to an impinging plane wave that propagates in direction $(\phi, \theta)$ is given by

$$
\begin{aligned}
S_{\mathrm{pw}}^{\text {surf }} & (\beta, \alpha, R, \omega) \\
& =\sum_{n=0}^{\infty} \sum_{m=-n}^{n} 4 \pi \mathrm{i}^{-n} Y_{n}^{m}(\phi, \theta)^{*} d_{n}(R, \omega) Y_{n}^{m}(\beta, \alpha) .
\end{aligned}
$$

The complex spherical surface harmonics $Y_{n}^{m}(\beta, \alpha)$ are defined as

$$
Y_{n}^{m}(\beta, \alpha)=(-1)^{m} \sqrt{\frac{2 n+1}{4 \pi} \frac{(n-|m|) !}{(n+|m|) !}} P_{n}^{|m|}(\cos \beta) \mathrm{e}^{-\mathrm{i} m \alpha} .
$$

$P_{n}^{|m|}(\cdot)$ are the associated Legendre functions [21]. $d_{n}(\cdot)$ is given by [3]

$$
d_{n}(R, \omega)=j_{n}\left(\omega \frac{R}{c}\right)-\frac{j_{n}^{\prime}\left(\omega \frac{R}{c}\right)}{h_{n}^{\prime(2)}\left(\omega \frac{R}{c}\right)} h_{n}^{(2)}\left(\omega \frac{R}{c}\right)
$$

and includes the effect of the scatterer. $j_{n}(\cdot)$ and $h_{n}^{(2)}(\cdot)$ denote the spherical Bessel and Hankel functions, respectively.

We equate (11) along the equator with the combination of (5) and (6) to match the sound field due to a horizontally propagating plane wave scattered off a spherical object to the sound field due to the same plane wave scattered off a cylinder with the same radius $R$. In the case of the cylinder, $\breve{S}_{m}(\omega)=\mathrm{i}^{-m} \mathrm{e}^{-\mathrm{i} m \theta}$ [21]. We also introduce modal correction weights $X_{m}(R, \omega)$ and obtain

$$
\begin{array}{r}
\sum_{n=0}^{\infty} \sum_{m=-n}^{n} 4 \pi \mathrm{i}^{-n} Y_{n}^{m}(\pi / 2, \theta)^{*} d_{n}(R, \omega) Y_{n}^{m}(\pi / 2, \alpha) \\
=\sum_{m=-\infty}^{\infty} \mathrm{i}^{-m} \mathrm{e}^{-\mathrm{i} m \theta} D_{m}(R, \omega) \mathrm{e}^{\mathrm{i} m \alpha} X_{m}(R, \omega) .
\end{array}
$$

We note that $Y_{n}^{m}(\beta, \alpha)=Y_{n}^{m}(\beta, 0) \mathrm{e}^{\mathrm{i} m \alpha}$ and use [8, Eq. (2.34)] to formulate (14) for each mode $m$, which yields

$$
\begin{aligned}
X_{m}(R, \omega) & =\frac{Z_{m}(R, \omega)}{D_{m}(R, \omega)} \\
= & \frac{\sum_{n=|m|}^{\infty} 4 \pi \mathrm{i}^{(m-n)}\left[Y_{n}^{m}(\pi / 2,0)\right]^{2} d_{n}(R, \omega)}{D_{m}(R, \omega)} .
\end{aligned}
$$

The dependency on the propagation direction $\theta$ cancels out.

Introducing (15) into (5) yields

$$
\breve{S}_{m}(\omega) D_{m}(R, \omega) X_{m}(R, \omega)=\stackrel{\circ}{m}_{m}^{\text {surf }}(R, \omega),
$$

which allows for computing the free-field CH coefficients $\breve{S}_{m}(\omega)$ of the incoming sound field $S(\vec{x}, \omega)$ as

$$
\breve{S}_{m}(\omega)=\frac{\stackrel{S}{S}_{m}^{\text {surf }}(R, \omega)}{Z_{m}(R, \omega)}=\frac{\int_{0}^{2 \pi} S^{\text {surf }}\left(\left.\vec{x}\right|_{r=R}, \omega\right) \mathrm{e}^{-\mathrm{i} m \alpha} \mathrm{d} \alpha}{2 \pi Z_{m}(R, \omega)} .
$$




\section{References}

[1] J. Meyer and G. Elko, "A highly scalable spherical microphone array based on an orthonormal decomposition of the soundfield," in IEEE ICASSP, 2002, pp. 1781-1784.

[2] T. D. Abhayapala and D. B. Ward, "Theory and design of high order sound field microphones using spherical microphone array," in IEEE ICASSP, 2002, pp. 1949-1952.

[3] B. Rafaely, "Plane-wave decomposition of the sound field on a sphere by spherical convolution," JASA, vol. 116, pp. 2149$2157,2004$.

[4] D. N. Zotkin, R. Duraiswami, and N. A. Gumerov, "Planewave decomposition of acoustical scenes via spherical and cylindrical microphone arrays," IEEE Transactions on Audio, Speech, and Language Processing, vol. 18, no. 1, pp. 2-16, 2010.

[5] B. Rafaely and A. Avni, "Interaural cross correlation in a sound field represented by spherical harmonics," JASA, vol. 127, no. 2, pp. 823-828, 2010.

[6] Z. Li and R. Duraiswami, "Headphone-based reproduction of $3 \mathrm{~d}$ auditory scenes captured by spherical/hemispherical microphone arrays," in IEEE ICASSP, 2006.

[7] Miguel Blanco Galindo, Philip Coleman, and Philip J.B. Jackson, "Microphone array geometries for horizontal spatial audio object capture with beamforming," JAES, vol. 68, no. 5, pp. 324-337, 2020.

[8] J. Ahrens, Analytic Methods of Sound Field Synthesis, Springer, Heidelberg, Germany, 2012.

[9] J. Blauert, Spatial Hearing: The Psychophysics of Human Sound Localization, The MIT Press, 101996.

[10] H. Teutsch and W. Kellermann, "Acoustic source detection and localization based on wavefield decomposition using circular microphone arrays," JASA, vol. 120, no. 5, pp. 2724-2736, 2006.

[11] A. Parthy, N. Epain, A. van Schaik, and C. T. Jin, "Comparison of the measured and theoretical performance of a broadband circular microphone array," JASA, vol. 130, no. 6, pp. 38273837, 2011.

[12] F. Kaiser, H. Pomberger, and . Zotter, "Investigations on cylindrical microphone arrays," in AmbisSym, York, UK, 2012.

[13] J. Trevino, S. Koyama, S. Sakamoto, and Y. Suzuki, "Mixedorder ambisonics encoding of cylindrical microphone array signals," Acoust. Sci. \& Tech., vol. 35, no. 3, pp. 174-177, 2014.

[14] T. Betlehem and M. Poletti, "Measuring the sphericalharmonic representation of a sound field using a cylindrical array," in IEEE ICASSP, 2019, pp. 955-959.

[15] J. Meyer, "Beamforming for a circular microphone array mounted on spherically shaped objects," JASA, vol. 109, 2001.

[16] E. Tiana-Roig, F. Jacobsen, and E. Fernandez-Grande, "Beamforming with a circular array of microphones mounted on a rigid sphere (L)," JASA, vol. 130, no. 3, pp. 1095-1098, 2011.
[17] M. Zaunschirm and F. Zotter, "Measurement-based modal beamforming using planar circular microphone arrays," in EAA Joint Symposium on Auralization and Ambisonics, Berlin, Germany, Apr. 2014, EAA, pp. 75-80.

[18] H. Chen, T. Abhayapala, and W. Zhang, "Theory and design of compact hybrid microphone arrays on two-dimensional planes for three-dimensional soundfield analysis," The Journal of the Acoustical Society of America, vol. 138, no. 5, pp. 3081-3092, 2015.

[19] S. Koyama, K. Furuya, K. Wakayama, S. Shimauchi, and H. Saruwatari, "Analytical approach to transforming filter design for sound field recording and reproduction using circular arrays with a spherical baffle," The Journal of the Acoustical Society of America, vol. 139, no. 3, pp. 1024-1036, 2016.

[20] V. R. Algazi, R. O. Duda, and D. M. Thompson, "Motiontracked binaural sound," JAES, vol. 52, no. 11, pp. 1142-1156, 2004.

[21] E. Williams, Fourier Acoustics: Sound Radiation and Nearfield Acoustical Holography, Academic Press, New York, 1999.

[22] J. Ahrens and S. Spors, "An analytical approach to sound field reproduction using circular and spherical loudspeaker distributions," Acta Acustica utd. with Acustica, vol. 94, no. 6, 2008.

[23] J. Ahrens and S. Spors, "Wave field synthesis of a sound field described by spherical harmonics expansion coefficients," JASA, vol. 131, no. 3, pp. 2190-2199, 32012.

[24] M. R. P. Thomas, J. Ahrens, and I. Tashev, "A method for converting between cylindrical and spherical harmonic representations of sound fields," in 2014 IEEE ICASSP, 2014, pp. 4723-4727.

[25] J. Ahrens and C. Andersson, "Perceptual evaluation of headphone auralization of rooms captured with spherical microphone arrays with respect to spaciousness and timbre," JASA, vol. 145, no. 4, pp. 2783-2794, 2019.

[26] "Sound Field Analysis toolbox for Python - Example 4," https://github.com/ AppliedAcousticsChalmers/sound_field_ analysis-py, Accessed: 2020-09-30.

[27] "Audio examples, ICASSP 2021," http: //www.ta.chalmers.se/research/ audio-technology-group/audio-examples/ icassp-2021/, Accessed: 2020-10-20.

[28] T. Lübeck, H. Helmholz, J. Arend, C. Pörschmann, and J. Ahrens, "Perceptual evaluation of mitigation approaches of impairments due to spatial undersampling in binaural rendering of spherical microphone array data," JAES, vol. 68, no. 6, pp. 428-440, 2020.

[29] J. Ahrens, H. Helmholz, D. Alon, and S. Amengual Garí, "Spherical Harmonic Decomposition of a Sound Field Based on Sensors Along the Equator of a Rigid Spherical Scatterer," JASA, 2021, (submitted). 